Pacific

Journal of

Mathematics

THE FINITE SIMPLE GROUPS HAVE COMPLEMENTED SUBGROUP LATTICES

Mauro Costantini And Giovanni Zacher

Volume $213 \quad$ No. 2

February 2004 


\title{
THE FINITE SIMPLE GROUPS HAVE COMPLEMENTED SUBGROUP LATTICES
}

\author{
Mauro Costantini and Giovanni Zacher
}

\begin{abstract}
We prove that the lattice of subgroups of every finite simple group is a complemented lattice.
\end{abstract}

\section{Introduction.}

A group $G$ is called a $K$-group (a complemented group) if its subgroup lattice is a complemented lattice, i.e., for a given $H \leq G$ there exists a $X \leq G$ such that $\langle H, X\rangle=G$ and $H \wedge X=1$. The main purpose of this Note is to answer a long-standing open question in finite group theory, by proving that:

\section{Every finite simple group is a K-group.}

In this context, it was known that the alternating groups, the projective special linear groups and the Suzuki groups are $K$-groups $([\mathbf{P}])$.

Our proof relies on the FSGC-theorem and on structural properties of the maximal subgroups in finite simple groups. The rest of this paper is divided into four sections. In Section 2 we collect some criteria for a subgroup of a group $G$ to have a complement and recall some useful known results. In Section 3 we deal with the classical groups, in 4 with the exceptional groups of Lie type and in Section 5 with the sporadic groups.

With reference to notation and terminology, we shall follow closely those in use in $[\mathbf{P}]$ and $[\mathbf{S}]$. All groups are meant to be finite.

\section{Preliminaries.}

We begin with the following:

Proposition 2.1. Given the group $G$, let $T, X$ be subgroups of $G$ such that $T \leq X<G$. If the interval $[X / T]$ is a complemented lattice and if $X$ is contained in only one maximal subgroup $M$ of $G$, then every $H \leq G$ with $H \not \leq M$ and $H \wedge T=1$ has a complement in $G$.

Proof. Let $C$ be a complement of $\langle H, T\rangle \wedge X$ in $[X / T]$. Then $\langle H, C\rangle=$ $\langle H, T, C\rangle \geq\langle\langle H, T\rangle \wedge X, C\rangle=X$. Since $H \not \leq M$, we conclude that $\langle H, C\rangle=$ $G$. Moreover $H \wedge C=H \wedge X \wedge C \leq\langle H, T\rangle \wedge X \wedge C=T$, hence $H \wedge C \leq$ $H \wedge T=1$. 
The condition on $M$ in Proposition 2.1 means that $[G / X]$ is a monocoatomic interval with coatom $M$.

Corollary 2.2. Let $X$ be a $K$-subgroup and $[G / X]$ a monocoatomic interval with coatom $M$. Then every $H \leq G$ not contained in $M_{G}$ has a complement in $G$. In particular $G / M_{G}$ is a $K$-group.

Proof. There exists a $g \in G$ such that $H^{g} \not \leq M$. By Proposition 2.1 with $T=$ $1, H^{g}$ has a complement. Hence also $H$ has a complement $C$ in $G$. Moreover, if $M_{G}<H$, then $C M_{G} / M_{G}$ is a complement of $H / M_{G}$ in $G / M_{G}$.

Proposition 2.3. Let $G$ be a simple group and $[G / X]$ a monocoatomic interval with coatom $M$. If $N$ is a central subgroup of $M$ of prime order with $N \leq X$ and if $X / N$ is a K-group, then $G$ is a $K$-group.

Proof. Let $H$ be a proper subgroup of $G$. Since $M_{G}=1$, without loss of generality we may assume $H \not \subset M$. If now $H \wedge N=1$, by Proposition 2.1 $H$ has a complement in $G$. Assume now $N \leq H$; there exists a $g \in G$ such that $N^{g} \wedge H=1$. So if $H$ has no complement in $G$, by Proposition 2.1 we must have $N^{g} \leq \mathcal{C}(H)$. It follows that if $\mathcal{F}=\left\{N^{x} \mid x \in G\right\}$ and $\mathcal{F}_{1}=\left\{N^{x} \mid\right.$ $\left.N^{x} \not \leq H\right\}$, then $\mathcal{N}(H) \geq\left\langle H, \mathcal{F}_{1}\right\rangle \geq\langle\mathcal{F}\rangle=G$, a contradiction.

We finally recall:

(2.1) The direct product of a family of groups is a K-group if and only if each factor is a K-group,

see Corollary 3.1 .5 in $[\mathbf{S}]$.

(2.2) If $G$ contains an abelian subgroup $A$ generated by minimal normal subgroups of $G$ and a complement $K$ to $A$ that is a $K$-group, then $G$ is a K-group,

see Lemma 3.1.9 in [S].

(2.3) The symmetric and alternating groups, the projective special linear groups $L_{n}(q)$ and the simple Suzuki groups ${ }^{2} B_{2}(q)$ are $K$-groups, see $[\mathbf{P}]$.

For our purpose it will be convenient to know which non-simple groups of Lie type $([\mathbf{C}]$, p. 175 , p. 268) are complemented.

Proposition 2.4. The following non-simple groups of Lie type are K-groups:

$$
L_{2}(2), L_{2}(3), S p_{4}(2), G_{2}(2),{ }^{2} G_{2}(3) .
$$

The following non-simple groups of Lie type are not $K$-groups:

$$
{ }^{2} B_{2}(2),{ }^{2} F_{4}(2), U_{3}(2) .
$$

Proof. In fact $L_{2}(2) \cong S_{3}, L_{2}(3) \cong A_{4}, S p_{4}(2) \cong S_{6}$, and we are done by (2.3). In $G_{2}(2)$ there is a monocoatomic interval $\left[G_{2}(2) / H\right]$ with $H \cong L_{3}(2)$ and corefree coatom, by Theorem 2.5 in $[\mathbf{C o}]$ : Hence $G_{2}(2)$ is a $K$-group by 
(2.3) and Corollary 2.2. The group ${ }^{2} G_{2}(3)$ has a corefree maximal subgroup isomorphic to $Z_{7}: Z_{6}([\mathbf{K} 3])$ : Hence it is a $K$-group by (2.2). On the other hand, we have ${ }^{2} B_{2}(2) \cong Z_{5}: Z_{4}([\mathbf{A}]), U_{3}(2) \cong 3^{2}: Q_{8}([\mathbf{K L}]$, p. 43$)$ and finally $\left|{ }^{2} F_{4}(2):{ }^{2} F_{4}(2)^{\prime}\right|=2$, but all involutions of ${ }^{2} F_{4}(2)$ are contained in ${ }^{2} F_{4}(2)^{\prime}([\mathbf{A S}]$, p. 75$)$.

To prove the main theorem, we take a counterexample $L$ of minimal order and show that such a group $L$ does not exist.

\section{The simple classical groups.}

We are going to assume in this section that $L=G_{0}(n, q)$, a (simple) classical group as in $[\mathbf{K L}]$.

a) $G_{0}(n, q)$ is not of type $A_{m}, n=m+1, m \geq 1$.

See (2.3).

b) $G_{0}(n, q)$ is not of type $C_{m}, n=2 m, m \geq 2$.

Proof. Let $r$ be a prime divisor of $m$, so that $m=r t, t \geq 1$. By Theorem 1 and Theorem 2 in $[\mathbf{L}]$, the interval $\left[P S p(2 m, q) / P S p\left(2 t, q^{r}\right)\right]$ is monocoatomic. Moreover $P S p\left(2 t, q^{r}\right)$ is simple, since $q^{r} \geq 4$, of order less than the order of $L$, hence a $K$-group. But then by Corollary 2.2, $L$ is a $K$-group, a contradiction.

c) $G_{0}(n, q)$ is not of type ${ }^{2} A_{m}, n=m+1, m \geq 2$.

Proof. We consider first the cases $(n, q)=(3,3),(3,5)$. The groups $U_{3}(3)$ and $U_{3}(5)$ are $K$-groups: In fact one has $P S L_{2}(7)<\cdot U_{3}(3)$ and $A_{7}<\cdot U_{3}(5)$ $([\mathbf{K} \mathbf{1}], \S 5)$. Assume now $(n, q) \neq(3,3),(3,5)$. With reference to the notation in [BGL], p. 388, let $G$ be the simple adjoint algebraic group over $\overline{\mathbb{F}}_{q}$ with associated Dynkin diagram of type $A_{m}, \lambda=\sigma_{q}$ and $\mu={ }^{2} \sigma_{q}$ : We have $G_{\lambda}=$ $P G L_{n}(q), G_{\mu}=P G U_{n}(q), O^{p^{\prime}}\left(G_{\lambda}\right)=L_{n}(q), O^{p^{\prime}}\left(G_{\mu}\right)=U_{n}(q)=G_{0}(n, q)$,

$$
T:=O^{p^{\prime}}\left(G_{\mu} \cap G_{\lambda}\right)= \begin{cases}P S p_{n}(q) & \text { if } n \text { is even } \\ \Omega_{n}(q) & \text { if } n q \text { is odd } \\ S p_{n-1}(q) & \text { if } n \text { is odd and } q \text { is even. }\end{cases}
$$

From Theorem 2 in $[\mathbf{B G L}]$ it follows that $\left[U_{n}(q) / T\right]$ is monocoatomic. Moreover, $T$ is a $K$-group, either because it is simple of order less than $|L|$, or because it is isomorphic to $S p_{4}(2)$ (Proposition 2.4): Hence $G_{0}(n, q)$ is a $K$-group, a contradiction.

d) $G_{0}(n, q)$ is not of type $B_{m}, n=2 m+1, m \geq 3, q$ odd. 
Proof. Assume $q=p^{f}$, with $f>1$ and let $r$ be a prime divisor of $f$. Then by Theorem 1 in $[\mathbf{B G L}],\left[P \Omega_{n}(q) / P \Omega_{n}\left(q^{1 / r}\right)\right]$ is monoatomic, a contradiction. Therefore we must have $q=p$. Now, by $\S 5$ in [K1] and Proposition 4.2.15 in $[\mathbf{K L}], G_{0}(n, q)$ contains a maximal subgroup $M$ which is a split extension of an irreducible elementary abelian 2-group by $A_{n}$ or $S_{n}$. Therefore $M$ is a $K$-group by (2.2), and $G_{0}(n, q)$ is a $K$-group, a contradiction.

e) $G_{0}(n, q)$ is not of type $D_{m}, n=2 m, m \geq 4$.

Proof. Let $V=\mathbb{F}_{q}^{n}$ be the natural (projective) module for $G_{0}(n, q)$, and let $W$ be a nonsingular subspace of $V$ of dimension 1 . Since $\bar{\Omega}:=G_{0}(n, q)$ is a counterexample of minimal order, the socle soc $H_{\bar{\Omega}}$ of the stabilizer $H_{\bar{\Omega}}$ of $W$ in $\bar{\Omega}$, which is isomorphic to $\Omega_{n-1}(q)$ if $q$ is odd, and to $S p_{n-2}(q)$ if $q$ is even, must be contained, by Corollary 2.2 , in an element $K_{\bar{\Omega}}$ of $\mathcal{C}(\bar{\Omega}) \cup \mathcal{S}$ different from $H_{\bar{\Omega}}$ (for the definition of the family $\mathcal{C}(\bar{\Omega}) \cup \mathcal{S}$ we refer to $\S 1.1$ and $\S 3.1$ in $[\mathbf{K L}])$.

By order considerations, one can prove that only condition $(i)$ of Theorem 4.2 in [Li] applies: This means that $K_{\bar{\Omega}}$ must be an element of $\mathcal{C}(\bar{\Omega})$. Since $H_{\bar{\Omega}} \in \mathcal{C}_{1}$, one is left to show that there does not exist an element $K_{\bar{\Omega}}$ in $\mathcal{C}_{i}$, for an $i \neq 1$, such that $\operatorname{soc} H_{\bar{\Omega}}<K_{\bar{\Omega}}<\bar{\Omega}$.

For $q$ odd, the arguments used in the proof of Proposition 7.1.3 in [KL] show that such a $K_{\bar{\Omega}}$ does not exist, taking into account that in our situation $n_{2}=n-1 \geq 7$. To deal with the case when $q$ is even, again one can proceed using arguments suggested in the proof of Lemma 7.1.4 in $[\mathbf{K L}]$.

f) $G_{0}(n, q)$ is not of type ${ }^{2} D_{m}, n=2 m, m \geq 4$.

Proof. Following the notation in $[\mathbf{B G L}]$, let $G$ be the simple adjoint algebraic group over $\overline{\mathbb{F}}_{q}$ with associated Dynkin diagram of type $D_{m}, \lambda=\sigma_{q}$ and $\mu={ }^{2} \sigma_{q}$. Then $O^{p^{\prime}}\left(G_{\lambda}\right)=P \Omega_{n}^{+}(q), O^{p^{\prime}}\left(G_{\mu}\right)=P \Omega_{n}^{-}(q)=G_{0}(n, q)$,

$$
T:=O^{p^{\prime}}\left(G_{\mu} \cap G_{\lambda}\right)= \begin{cases}\Omega_{n-1}(q) & \text { if } q \text { is odd } \\ S p_{n-2}(q) & \text { if } q \text { is even. }\end{cases}
$$

By Theorem 2 in $[\mathbf{B G L}],\left[G_{0}(n, q) / T\right]$ is monocoatomic. Since $n \geq 8, T$ is simple, hence $G_{0}(n, q)$ is a $K$-group, a contradiction.

We have therefore completed the proof that $L$ is not a classical group.

\section{The simple exceptional groups of Lie type.}

Now we are going to show that the minimal counterexample $L$ cannot be an exceptional group of Lie type $G(q)$.

a) $G(q)$ is not of type $G_{2},{ }^{2} G_{2}$. 
Proof. If $r$ is a prime divisor of $f$, where $q=p^{f}$, write $q=q_{0}^{r}$. Then $G\left(q_{0}\right)<\cdot G(q)$ ([Co], Theorem 2.3, 2.4, [K3], Theorem A, C). Hence by Proposition 2.4, we have $L=G_{2}(p)$, for an odd prime $p$. But then $G_{2}(2)$ is maximal in $G_{2}(p)$ by $[\mathbf{K 3}]$, and we are done by Proposition 2.4.

\section{b) $G(q)$ is not of type $F_{4}$.}

Proof. $F_{4}(q)$ contains a quasisimple maximal subgroup $M$ of type $B_{4}(q)$, with $|Z(M)|=(2, q-1)$ ([LSS $]$, p. 322). But then, by Proposition 2.3, $F_{4}(q)$ is a $K$-group.

c) $G(q)$ is not of type $E_{6}, E_{7}, E_{8}$.

Proof. We have $F_{4}(q)<\cdot E_{6}(q)\left([\mathbf{L S}]\right.$, Table 1), which excludes $E_{6}$.

If $L$ is of type $E_{7}$, there exist subgroups $H \leq M<\cdot G$ such that $|M: H|=$ $|Z(H)|=(2, q-1)$ and $H / Z(H) \cong L_{2}(q) \times P \Omega_{12}^{+}(q)([\mathbf{L S}]$, Table 1). Hence $H / Z(H)$ is a $K$-group by $(2.1)$. We claim that $[G / H]$ is monocoatomic. Clear if $q$ is even. For $q$ odd, suppose $H<M_{1}<\cdot G$, with $M_{1} \neq M$. Since $|M: H|=2$, we have $\left|M_{1}\right|>|M| \geq q^{64}$. By the Theorem in [LS], $M_{1}$ either is a parabolic subgroup, or it appears in Table 1 in [LS]: However, both situations are excluded by rank or order considerations. So again by Proposition 2.3, $G$ is a $K$-group, a contradiction.

Finally assume $G$ is of type $E_{8}$. There exist subgroups $H \leq M<\cdot G$ such that $|M: H|=|Z(H)|=(2, q-1)$, with $H / Z(H) \cong P \Omega_{16}^{+}(q)([\mathbf{I}]$, p. 286, [LS], Table 1$)$, hence a $K$-group. Using the Theorem in $[\mathbf{L S}]$ again one shows that $[G / H]$ is monoatomic, giving rise to a contradiction.

d) $G(q)$ is not of type ${ }^{2} B_{2}$.

See (2.3).

e) $G(q)$ is not of type ${ }^{2} F_{4}$.

Proof. The group ${ }^{2} F_{4}(2)$ is not simple, and we have seen that it is not a $K$ group (Proposition 2.4). Its derived subgroup (the Tits group) is simple and it is a $K$-group, since it has a maximal subgroup isomorphic to $L_{2}(25)([\mathbf{A}])$. So now assume $L={ }^{2} F_{4}\left(2^{2 m+1}\right)$, with $m \geq 1$. By the Main Theorem in [M], there exist $H<\cdot M<\cdot L$ such that $|M: H|=2$ and $H \cong S p_{4}\left(2^{2 m+1}\right)$. Since the nonabelian composition factors of maximal subgroups of $L$ not conjugate to $M$ are of type $A_{1}(q),{ }^{2} B_{2}(q), U_{3}(q)$ and ${ }^{2} F_{4}\left(q^{1 / r}\right), r$ an odd prime, one concludes that $[G / H]$ is monocoatomic.

f) $G(q)$ is not of type ${ }^{2} E_{6}$.

Proof. In fact we have $F_{4}(q)<\cdot{ }^{2} E_{6}(q)$ from Table 1 in $[\mathbf{L S}]$. 
g) $G(q)$ is not of type ${ }^{3} D_{4}$.

Proof. From the Theorem in $[\mathbf{K 2}]$, we have $G_{2}(q)<\cdot{ }^{3} D_{4}(q)$. Since $G_{2}(q)$ is a $K$-group, we get a contradiction.

This concludes the proof that $L$ is not a group of Lie type.

\section{Sporadic simple groups.}

We are left to deal with the sporadic groups: To this end, for each group we exhibit a maximal subgroup which is a $K$-group. From the tables in $[\mathbf{A}]$ we have:

$$
\begin{aligned}
& L_{2}(11)<\cdot M_{11}, \quad L_{2}(11)<\cdot M_{12}, \quad A_{7}<\cdot M_{22}, \quad M_{22}<\cdot M_{23}, \quad M_{23}<\cdot M_{24}, \\
& L_{2}(11)<\cdot J_{1}, \quad A_{5}<\cdot J_{2}, \quad L_{2}(19)<\cdot J_{3}, \quad 43: 14<\cdot J_{4}, \quad M_{22}<\cdot H S, \\
& A_{7}<\cdot S u z, \quad M_{22}<\cdot M c L, \quad A_{8}<\cdot R u, \quad S_{4} \times L_{3}(2)<\cdot H e, \quad 67: 22<\cdot L y, \\
& A_{7}<\cdot O^{\prime} N, \quad M_{23}<\cdot C o_{2}, \quad M_{23}<\cdot C o_{3}, \quad C o_{3}<\cdot C o_{1}, \quad S_{10}<\cdot F i_{22}, \\
& S_{12}<\cdot F i_{23}, \quad F i_{23}<\cdot F i_{24}^{\prime}, \quad A_{12}<\cdot H N, \quad S_{5}<\cdot T h, \quad 31: 15<\cdot B M, \\
& 31: 15 \times S_{3}<\cdot M .
\end{aligned}
$$

We have thus completed the proof of the main theorem:

Theorem. Every finite simple group is a K-group.

Acknowledgements. We are grateful to E. Vdovin for helpful discussions.

\section{References}

[AS] M. Aschbacher and G. Seitz, Involutions in Chevalley groups, Nagoya Math. J., 63 (1976), 1-91, MR 0422401, Zbl 0359.20014.

[BGL] N. Burgoyne, R. Griess and R. Lyons, Maximal subgroups and automorphisms of Chevalley groups, Pac. J. Math., 71 (1977), 365-403, MR 0444795, Zbl 0334.20022.

[C] R.W. Carter, Simple Groups of Lie Type, Wiley, London, 1989, MR 1013112, Zbl 0723.20006.

[A] J.H. Conway, R.T. Curtis, S.P. Norton, R.A. Parker and R.A. Wilson, Atlas of Finite Groups, Oxford University Press, 1985, MR 0827219, Zbl 0568.20001.

[Co] B.N. Cooperstein, Maximal subgroups of $G_{2}\left(2^{n}\right)$, J. Algebra, 70 (1981), 23-36, MR 0618376, Zbl 0459.20007.

[I] N. Iwahori, Centralizers of involutions in finite Chevalley groups, in 'Seminar on Algebraic Groups and Related Topics' (eds. A. Borel et al.), Lecture Notes in Math., 131, Springer, Berlin, 1986, 267-295, MR 0258945, Zbl 0242.20043.

[K1] P.B. Kleidman, The Low-Dimensional Finite Classical Groups and their Subgroups, Ph.D. Thesis, University of Cambridge, 1987. 
[K2] - The maximal subgroups of the Steinberg triality groups ${ }^{3} D_{4}(q)$ and of their automorphism groups, J. Algebra, 115 (1988), 182-199, MR 0937609, Zbl 0642.20013.

[K3] - The maximal subgroups of the Chevalley groups $G_{2}(q)$ with $q$ odd, the Ree groups ${ }^{2} G_{2}(q)$, and their automorphism groups, J. Algebra, 117 (1988), 30-71, MR 0955589, Zbl 0651.20020.

[KL] P.B. Kleidman and M.W. Liebeck, The Subgroup Structure of the Finite Classical Groups, London Math. Soc. Lecture Notes, 129, Cambridge University Press, 1990, MR 1057341, Zbl 0697.20004.

[L] S. Li, Overgroups in $G L(n r, F)$ of certain subgroups of $S L(n, K)$, I, J. Algebra, 125 (1989), 215-235, MR 1012672, Zbl 0676.20030.

[Li] M.W. Liebeck, On the orders of maximal subgroups of the finite classical groups, Proc. London Math. Soc., 50 (1985), 426-446, MR 0779398, Zbl 0591.20021.

[LS] M.W. Liebeck and J. Saxl, On the orders of maximal subgroups of the finite exceptional groups of Lie type, Proc. London Math. Soc., 55 (1987), 299-330, MR 0896223, Zbl 0627.20026.

[LSS] M.W. Liebeck, J. Saxl and G.M. Seitz, Subgroups of maximal rank in finite exceptional groups of Lie type, Proc. London Math. Soc., 65 (1992), 297-325, MR 1168190, Zbl 0776.20012.

[M] G. Malle, The maximal subgroups of ${ }^{2} F_{4}\left(q^{2}\right)$, J. Algebra, 139 (1991), 52-69, MR 1106340, Zbl 0725.20014.

[P] E. Previato, Some families of simple groups whose lattices are complemented, Boll. U.M.I., 6 (1982), 1003-1014, MR 0683488.

[S] R. Schmidt, Subgroup Lattices of Groups, de Gruyter, Berlin, 1994, MR 1292462, Zbl 0843.20003.

Received December 24, 2002 and revised February 24, 2003.

DiPARTIMENTO Di MATEMATICA PURA ED APPLICATA

UNIVERSiTÀ DI PADOVA

VIA Belzoni 7, 35131 PAdova

ITALY

E-mail address: costantini@math.unipd.it

DiPARTIMENTO Di MATEMATICA PURA ED APPLICATA

Università Di PADOVA

VIA Belzoni 7, 35131 PAdova

ITALY

E-mail address: zacher@math.unipd.it 